\title{
The interaction of oxygen with small gold clusters
}

\author{
Sergey A. Varganov, Ryan M. Olson, and Mark S. Gordon \\ Department of Chemistry, Iowa State University, Ames, Iowa 50011 \\ Horia Metiua) \\ Department of Chemistry and Biochemistry, University of California-Santa Barbara, Santa Barbara, \\ California 93106
}

(Received 10 December 2002; accepted 7 May 2003)

\begin{abstract}
Presented in this work are the results of a quantum chemical study of oxygen adsorption on small $\mathrm{Au}_{n}$ and $\mathrm{Au}_{n}^{-}(n=2,3)$ clusters. Density functional theory (DFT), second order perturbation theory (MP2), and singles and doubles coupled cluster theory with perturbative triples [CCSD $(\mathrm{T})]$ methods have been used to determine the geometry and the binding energy of oxygen to $\mathrm{Au}_{n}$. The multireference character of the wave functions has been studied using the complete active space self-consistent field method. There is considerable disagreement between the oxygen binding energies provided by CCSD $(\mathrm{T})$ calculations and those obtained with DFT. The disagreement is often qualitative, with DFT predicting strong bonds where $\operatorname{CCSD}(\mathrm{T})$ predicts no bonds or structures that are bonded but have energies that exceed those of the separated components. The CCSD(T) results are consistent with experimental measurements, while DFT calculations show, at best, a qualitative agreement. Finally, the lack of a regular pattern in the size and the sign of the errors [as compared to $\operatorname{CCSD}(\mathrm{T})$ ] is a disappointing feature of the DFT results for the present system: it is not possible to give a simple rule for correcting the DFT predictions (e.g., a useful rule would be that DFT predicts stronger binding of $\mathrm{O}_{2}$ by about $0.3 \mathrm{eV}$ ). It is likely that the errors in DFT appear not because of gold, but because oxygen binding to a metal cluster is a particularly difficult problem.

(C) 2003 American Institute of Physics. [DOI: 10.1063/1.1587115]
\end{abstract}

\section{INTRODUCTION}

Recent experiments have shown that small gold clusters deposited on a metal oxide surface are good oxidation catalysts. ${ }^{1}$ This has stimulated theoretical work on oxygen adsorption in the gas-phase, ${ }^{2}$ on oxide-supported $\mathrm{Au}$ clusters, ${ }^{3}$ and on the mechanism of $\mathrm{CO}$ oxidation catalyzed by gold. ${ }^{4}$ These papers used density functional theory (DFT) to calculate the binding energies and the activation energies needed for clarifying the reaction mechanism. Most catalytic systems have many electrons, making it challenging, but not impossible given new linear scaling methods, ${ }^{5}$ to employ more reliable and more accurate methods.

When using DFT it is implicitly assumed that the calculated energies are sufficiently accurate for the task at hand. In most cases it is difficult to assess the accuracy of DFT methods, since the experimental data needed for such tests are not available. Moreover, the theory is particularly valuable when it provides information about aspects of a reaction that cannot be obtained by experiments (e.g., the transition state, transient reaction intermediates). In such cases, the reliability of the results given by the DFT calculations must be determined without appeal to experiments.

One approach to testing the accuracy of DFT methods is to compare the results to those provided by better methods. To accomplish this one must study "model systems" that are small enough to allow the use of accurate methods, and large

${ }^{a)}$ Electronic mail: metiu@chem.ucsb.edu enough to contain all the significant aspects of the system of interest.

This article presents calculations of the binding energies of one or two oxygen molecules to $\mathrm{Au}_{2}$ and $\mathrm{Au}_{3}$ neutral and negatively charged clusters. To test the accuracy of the DFT calculations, energy differences based on the B3LYP ${ }^{6}$ and PW $91^{7}$ functionals are compared with those obtained using second-order perturbation theory $(\mathrm{MP} 2)^{8}$ and singles and doubles coupled cluster theory with perturbative triples $[\operatorname{CCSD}(\mathrm{T})],{ }^{9}$ as well as with multireference methods. The multireference approaches ensure that a correct zeroth-order wave function is used, while the perturbation and (especially) coupled cluster methods provide a correct accounting of dynamic correlation.

In assessing the practical importance of the findings obtained in the present work, it is important to recognize that the key issue in catalysis studies is to understand the reaction mechanisms that lead to products via pathways that have the smallest activation energy. For example, in the case of $\mathrm{CO}$ oxidation one can envision two mechanisms: in one $\mathrm{O}_{2}$ dissociates and an $\mathrm{O}$ atom reacts with $\mathrm{CO}$ producing $\mathrm{CO}_{2}$; in the other $\mathrm{O}_{2}$ reacts with $\mathrm{CO}$ to form a carbonate and this reacts with $\mathrm{CO}$ to produce two $\mathrm{CO}_{2}$ molecules. One can decide between the two possibilities by finding which one has the lowest activation energy. A theoretical prediction is useful if it is sufficiently accurate to tell which of the two activation energies is smaller. The same can be said about finding the most likely reaction intermediate from a set of candidates. DFT calculations could be very useful, if they are accurate enough for the tasks described earlier. 
TABLE I. Bond distances $R(\AA)$, dissociation energies $D_{e}(\mathrm{eV})$, and vibrational frequencies $\omega_{e}(1 / \mathrm{cm})$ of $A u_{2}$ at different levels of theory.

\begin{tabular}{|c|c|c|c|c|c|c|c|c|c|c|c|c|}
\hline & \multicolumn{3}{|c|}{$\operatorname{SBKJC}(f)+s p$} & \multicolumn{3}{|c|}{$\operatorname{uSBKJC}(f)+s p$} & \multicolumn{3}{|c|}{$\mathrm{uSBKJC}(3 f)+s p$} & \multicolumn{3}{|c|}{$\mathrm{uSBKJC}(3 f 2 g)+s p$} \\
\hline & $R(\AA)$ & $D_{e}(\mathrm{eV})$ & $\omega_{e}(1 / \mathrm{cm})$ & $R(\AA)$ & $D_{e}(\mathrm{eV})$ & $\omega_{e}(1 / \mathrm{cm})$ & $R(\AA)$ & $D_{e}(\mathrm{eV})$ & $\omega_{e}(1 / \mathrm{cm})$ & $R(\AA)$ & $D_{e}(\mathrm{eV})$ & $\omega_{e}(1 / \mathrm{cm})$ \\
\hline $\mathrm{HF}$ & 2.615 & 0.80 & 156 & 2.612 & 0.83 & 156 & 2.605 & 0.86 & 156 & 2.598 & 0.88 & 156 \\
\hline MP2 & 2.494 & 2.24 & 190 & 2.472 & 2.28 & 194 & 2.469 & 2.35 & 196 & 2.448 & 2.47 & 200 \\
\hline CCSD & 2.539 & 1.89 & $\mathrm{e}$ & 2.521 & 1.89 & $\mathrm{e}$ & 2.527 & 1.87 & $\mathrm{e}$ & 2.509 & 1.94 & $\mathrm{e}$ \\
\hline $\operatorname{CCSD}(\mathrm{T})$ & 2.535 & 2.04 & 175 & 2.517 & 2.07 & $\mathrm{e}$ & 2.523 & 2.06 & $\mathrm{e}$ & 2.504 & 2.14 & e \\
\hline $\operatorname{CASSCF}(2,2)$ & 2.654 & 1.12 & 136 & e & $\mathrm{e}$ & $\mathrm{e}$ & e & e & e & e & e & e \\
\hline MRMP2(2,2) & 2.506 & 2.17 & 184 & 2.483 & 2.22 & $\mathrm{e}$ & 2.488 & 2.23 & e & 2.465 & 2.35 & e \\
\hline B3LYP & 2.562 & 1.98 & 167 & 2.560 & 1.97 & 167 & 2.547 & 2.01 & 167 & 2.539 & 2.03 & 168 \\
\hline PW-PBE ${ }^{\mathrm{a}}$ & 2.54 & 2.22 & & & & & & & & & & \\
\hline PW-PW91 & 2.528 & 2.27 & & & & & & & & & & \\
\hline $\mathrm{cp}-\operatorname{CCSD}(\mathrm{T})^{\mathrm{c}}$ & 2.488 & 2.19 & 187 & & & & & & & & & \\
\hline Experiment ${ }^{\mathrm{d}}$ & 2.472 & 2.31 & 191 & & & & & & & & & \\
\hline
\end{tabular}

${ }^{a}$ DFT with plane-wave basis set and PBE functional [Ref. 4(a)].

${ }^{\mathrm{b}}$ DFT with plane-wave basis set and PW91 functional (Ref. 2).

${ }^{c}$ Counterpose-corrected CCSD(T) with all electron PJHN-4f2g1h1i basis set (Ref. 28).

${ }^{\mathrm{d}}$ Experimental values (Ref. 29).

${ }^{\mathrm{e}}$ Values are not available yet.

\section{COMPUTATIONAL METHODS}

For all systems studied here, the geometries were first determined using Hartree-Fock (HF) calculations. To ensure that the optimized geometries correspond to minima on their respective potential energy surfaces, the Hessian (matrix of energy second derivatives) was calculated and diagonalized. Hessians that are positive definite (no negative eigenvalues) correspond to local minima. Those with $n$ negative eigenvalues correspond to $n$th order saddle points. The optimized HF geometries were used as the initial structures for DFT and MP2 geometry optimizations. The optimized MP2 geometries were then used for single point $\operatorname{CCSD}(\mathrm{T})$ calculations. The energy gradients for geometry optimizations were evaluated analytically for all HF, DFT, and closed shell MP2 calculations. For open-shell MP2 geometry optimizations, the gradients were calculated numerically.

To avoid spin contamination in the wave function, restricted open-shell methods were used for all open shell calculations. For open-shell second-order perturbation theory, the $Z$-averaged perturbation theory $\left(\mathrm{ZAPT}^{10}\right)$ version of restricted MP2 was used. The ZAPT and the restricted $\operatorname{RCCSD}(\mathrm{T})^{11}$ method are based on a restricted open-shell HF wave function that is free of spin contamination. In the MP2 and $\operatorname{CCSD}(\mathrm{T})$ calculations the $1 s$ orbitals of oxygen and $5 s$ and $5 p$ core orbitals of gold atoms were not included in the correlation part of the calculation.

To study the importance of nondynamic electron correlation, and to assess whether the single determinant-based methods provide an acceptable zeroth-order wave function in the small gold clusters, complete active space self-consistent field $(\mathrm{CASSCF})^{12}$ and multireference second-order perturbation theory $(\mathrm{MRMP} 2)^{13}$ calculations were carried out on $\mathrm{Au}_{2}$ and $\mathrm{Au}_{3}$ clusters.

The restricted DFT method with the B3LYP functional was used to compare with the results of previous plane wavebased DFT calculations ${ }^{2}$ using the PW91 functional and with the MP2, MRMP2, and CCSD(T) calculations described ear- lier. The B3LYP functional consists of five functionals, namely Becke + Slater + HF exchange and LYP + VWN5 correlation.

In all calculations the effective core potential (ECP) with scalar relativistic corrections developed by Stevens et al. $(\mathrm{SBKJC})^{14}$ was used for the gold atoms. This ECP retains 19 explicit electrons. The Gaussian contracted SBKJC basis set $^{13}$ was augmented with one $f$ polarization (exponent $=0.89$ ) and one $s$ and one $p$ diffuse function (both exponents $=0.01)$ centered on the gold atoms. The standard contracted $6-31+\mathrm{G}^{*}$ basis set was centered on each oxygen atom. In all calculations, spherical harmonic basis functions were used with five $d$ orbitals and seven $f$ orbitals. In order to study the effect of the contraction of the basis set on the computational results, calculations on $\mathrm{Au}_{2}$ were also carried out with uncontracted basis sets.

The GAMESS ${ }^{15}$ suite of programs was used for all calculations except for $\operatorname{CCSD}(\mathrm{T})$, which was done in MOLPRO. ${ }^{16}$

\section{RESULTS AND DISCUSSION}

\section{A. Results for $\mathrm{Au}_{2}$}

In Table I, we show the bond length, the binding energy, and the vibrational frequency of $\mathrm{Au}_{2}$, calculated with a variety of methods and basis sets, as well as the experimental results. The starting basis set is the standard SBKJC ECP, augmented by a set of $f$ functions and a set of diffuse $s$ and $p$ functions. Several observations about Table I are pertinent. (a) As one might expect, compared with the experimental values, the bond distances predicted by HF are much too large, the dissociation energies much too small, and the vibrational frequency too small. These observations are essentially independent of basis set. (b) The B3LYP results are considerably better than those obtained using HF, they are essentially independent of basis set (suggesting that these results are converged with basis set), the dissociation energy is still about $0.3 \mathrm{eV}$ too small, and the vibrational frequency 
TABLE II. $\mathrm{Au}_{2}$ vertical and adiabatic electron affinities $(\mathrm{eV})$ calculated with different levels of theory. Experimental values for the adiabatic electron affinity are $1.94 \mathrm{eV}$ (Ref. 17) and $2.02 \mathrm{eV}$ (Ref. 18). PW-PW91 (Ref. 2) and PW-PBE [Ref. 4(a)] values for adiabatic electron affinity are 2.02 and $2.08 \mathrm{eV}$. Relativistic CCSD(T) with all electron $(10 s 7 p 5 d 3 f) /[10 s 6 p 5 d 3 f]$ basis set value is $1.83 \mathrm{eV}$ (Ref. 21).

\begin{tabular}{llcccccc}
\hline \hline & & HF & MP2 & CCSD(T) & CASSCF(2,2) & MRMP2 & B3LYP \\
\hline Contracted & Vertical & 0.58 & 1.43 & 1.46 & 0.33 & 1.49 & 1.66 \\
basis set & Adiabatic & 0.64 & 1.47 & 1.51 & 0.36 & 1.52 & 1.71 \\
Uncontracted & Vertical & 0.59 & 1.48 & 1.51 & 0.34 & 1.54 & 1.68 \\
basis set & Adiabatic & 0.65 & 1.53 & 1.56 & 0.37 & 1.58 & 1.74 \\
\hline \hline
\end{tabular}

is only marginally better than the HF value. (c) The MP2 results are in surprisingly good agreement with experiment, but this is clearly an artifact of the cancellation of basis set errors and level-of-theory errors since the agreement deteriorates as the basis set is improved. (d) The $\operatorname{CCSD}(\mathrm{T})$ results, with the smallest basis set, are already better than DFT and, unlike MP2, steadily improve as the basis set is improved. (e) Uncontracting the ECP valence basis set has little effect, and the same may be said of the multireference methods, CASSCF and MRMP2. Previous plane wave DFT calculations $^{2}$ also predict reasonable dissociation energies, but these calculations are not expected to be as accurate as $\operatorname{CCSD}(\mathrm{T})$ in general.

Table II contains the values of vertical (anion has the neutral geometry) and adiabatic (optimal geometries for both neutral and anion) electron affinities (EA) of $\mathrm{Au}_{2}$ dimer, calculated with different methods using contracted and completely uncontracted basis sets. The experimental adiabatic EA has been estimated to be $1.94 \mathrm{eV}{ }^{17}$ Taylor et al. estimate the EA to be $2.02 \mathrm{eV}^{18}{ }^{18}$ Mills, Gordon, and Metiu ${ }^{2}$ calculate an adiabatic EA of $2.02 \mathrm{eV}$ using plane waves and the PW91 functional, and Hakkinen and Landman, ${ }^{4(a)}$ using the PBE functional, ${ }^{19}$ predict $2.08 \mathrm{eV}$. Turning to Table II, it is clear that, as was noted for the results in Table I, uncontracting the basis set does not significantly improve the results. Of course, this only expands the underlying $s, p$ basis. The HF and CASSCF results are again unsatisfactory. All other methods are in much better agreement with experiment, although they all underestimate the experimental value of $1.94 \mathrm{eV}$ by $0.2-0.4 \mathrm{eV}$. However, based on the results in Table I, it is expected that an analogous basis set improvement, by adding more and higher $l$ polarization functions, will bring the $\operatorname{CCSD}(\mathrm{T})$ results into much better agreement with experiment, while having little effect on the DFT predictions. We also note that both PW91 and PBE calculations are in excellent agreement with the experiments.

\section{B. Results for $\mathrm{Au}_{3}$}

The $D_{3 h}$ geometry of the $\mathrm{Au}_{3}$ cluster has one electron in the doubly degenerate highest occupied molecular orbital, so it is subject to Jahn-Teller distortion. The distorted $\mathrm{Au}_{3}$ cluster has $C_{2 v}$ symmetry and two possible electronic states, ${ }^{2} A_{1}$ and ${ }^{2} B_{2}$ (Fig. 1). The energy relative to the ${ }^{2} B_{2}$ state and the geometry of the $\mathrm{Au}_{3}$ cluster are shown in Table III. According to experiment, ${ }^{20}$ the ground state of $\mathrm{Au}_{3}$ is ${ }^{2} B_{2}$, and the energy splitting between the ${ }^{2} A_{1}$ and the ${ }^{2} B_{2}$ states is very small. Wesendrup et al. ${ }^{21}$ showed that even relativistic
$\operatorname{CCSD}(\mathrm{T})$ calculations with large basis sets predict the ${ }^{2} A_{1}$ state to be the ground state with the energy splitting between it and the ${ }^{2} B_{2}$ state of $4.4 \mathrm{kcal} / \mathrm{mol}$. The same authors showed that relativistic MP2 calculations predict the ${ }^{2} B_{2}$ state to be $0.9 \mathrm{kcal} / \mathrm{mol}$ more stable than ${ }^{2} A_{1}$. The last result is consistent with the order of the states predicted by the multireference singles and doubles configuration interaction $\left(\right.$ MRSDCI $^{22}$ ) method. ${ }^{23}$

The HF and MRMP2 methods predict, respectively, that the ${ }^{2} A_{1}$ state is 1.1 and $0.1 \mathrm{kcal} / \mathrm{mol}$ lower in energy than the ${ }^{2} B_{2}$ state. However, the CASSCF and MP2 methods predict that the ${ }^{2} B_{2}$ state is lower than ${ }^{2} A_{1}$ by 1.9 and $1.0 \mathrm{kcal} / \mathrm{mol}$, respectively. Interestingly, the B3LYP method and the planewave PW91 calculations predict no Jahn-Teller distortion: the linear ${ }^{2} \Sigma_{u}^{+}$is the lowest energy state. These results indicate that larger basis sets and consideration of the spin-orbit interaction among the states is required for an accurate prediction of the order of the $\mathrm{Au}_{3}$ electronic states. Since the energy difference between the ${ }^{2} B_{2}$ and ${ }^{2} A_{1}$ states is very small, in the following calculations of the binding energy of oxygen molecules to the $\mathrm{Au}_{3}$ cluster, it is assumed that ${ }^{2} B_{2}$ is the ground state.

\section{Results for $\mathrm{Au}_{3}^{-}$}

All methods find that the $\mathrm{Au}_{3}^{-}$cluster is linear, although local minima are also found in $C_{2 v}$ and $D_{3 h}$ symmetry. The energies of the $C_{2 v}{ }^{1} A_{1}$ and the $D_{3 h}{ }^{3} A_{1}$ states relative to linear ${ }^{1} \Sigma_{g}^{+}$are shown in Table IV. All methods predict that the ${ }^{3} A_{1}$ and ${ }^{1} A_{1}$ states are approximately 20 and $40 \mathrm{kcal} / \mathrm{mol}$, respectively, higher than ${ }^{1} \Sigma_{g}^{+}$. However, the methods that include dynamic correlation [MP2, CCSD(T) and MRMP2] all predict significantly shorter distances for all isomers than the other methods (Fig. 2).

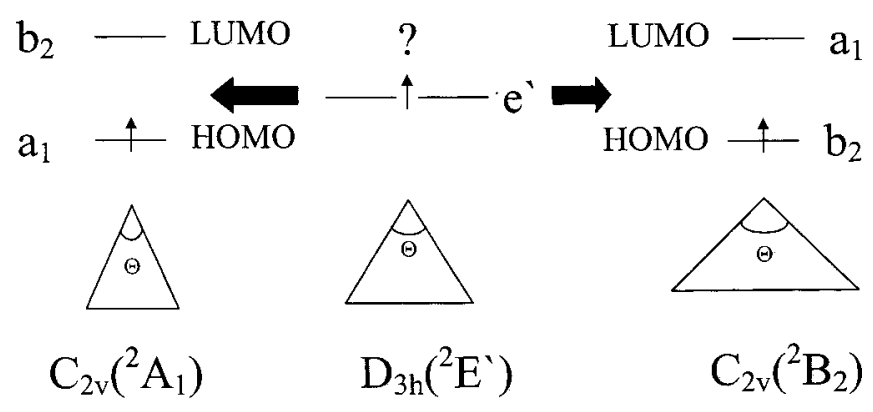

FIG. 1. Jahn-Teller distortion in $\mathrm{Au}_{3}$ cluster. Highest occupied (HOMO) and lowest unoccupied (LUMO) molecular orbitals of $\mathrm{Au}_{3}$ are shown. 
TABLE III. Bond distances $R(\AA)$, angles $\Theta(\mathrm{deg})$ and energies $\Delta E(\mathrm{kcal} / \mathrm{mol})$ relative to ${ }^{2} B_{2}$ state of $\mathrm{Au}_{3}$ at different levels of theory.

\begin{tabular}{|c|c|c|c|c|c|c|c|}
\hline & \multicolumn{2}{|c|}{${ }^{2} B_{2}$} & \multicolumn{3}{|c|}{${ }^{2} A_{1}$} & \multicolumn{2}{|c|}{${ }^{2} \Sigma_{u}^{+}$} \\
\hline & $R$ & $\Theta$ & $\Delta E$ & $R$ & $\Theta$ & $\Delta E$ & $R$ \\
\hline HF & 2.711 & 71.8 & -1.1 & 2.983 & 52.6 & 1.4 & 2.701 \\
\hline MP2 & 2.580 & 64.3 & 1.0 & 2.688 & 56.3 & 6.5 & 2.558 \\
\hline $\operatorname{CCSD}(\mathrm{T})$ & $M P 2^{d}$ & MP2 & 0.3 & MP2 & MP2 & 5.0 & MP2 \\
\hline $\operatorname{CASSCF}(3,3)$ & 2.749 & 143.5 & 1.9 & 3.031 & $52.2 \mathrm{TS}^{\mathrm{c}}$ & 0.0 & 2.755 \\
\hline MRMP2 & 2.591 & 64.9 & -0.1 & 2.681 & 56.8 & 34.8 & 2.629 \\
\hline RB3LYP & 2.649 & 68.6 & 0.7 & 2.945 & 52.2 & -0.5 & 2.628 \\
\hline UB3LYP & 2.650 & 68.5 & 0.7 & 2.793 & 55.7 & -0.5 & 2.630 \\
\hline $\mathrm{R}-\operatorname{CCSD}(\mathrm{T})^{\mathrm{a}}$ & 2.607 & 65.4 & -4.4 & 2.723 & 56.2 & 58.4 & 2.573 \\
\hline $\mathrm{R}-\mathrm{MP} 2^{\mathrm{a}}$ & 2.552 & 65.1 & 0.9 & 2.665 & 56.1 & 3.2 & 2.517 \\
\hline $\mathrm{MRSDCI}^{\mathrm{b}}$ & 2.60 & 65.7 & 0.6 & 2.72 & 56.4 & $\cdots$ & $\cdots$ \\
\hline
\end{tabular}

${ }^{\mathrm{a}}$ Relativistic $\operatorname{CCSD}(\mathrm{T})$ and MP2 with all electron $(10 s 7 p 5 d 3 f) /[10 s 6 p 5 d 3 f]$ basis set (Ref. 21).

${ }^{b}$ Multireference single and double configuration interaction with Davidson correction [Ref. 23(a)].

${ }^{\mathrm{c}}$ This is a transition state on the potential energy surface.

${ }^{\mathrm{d}}$ MP2 geometries.

\section{Results for $\mathrm{Au}_{2} \mathrm{O}_{2}$ and $\mathrm{Au}_{2}\left(\mathrm{O}_{2}\right)_{2}$}

The binding energies of the first and second oxygen molecules to the neutral gold clusters were determined from

$$
\begin{aligned}
& E_{b 1}=E\left[\mathrm{Au}_{n}\right]+E\left[\mathrm{O}_{2}\right]-E\left[\mathrm{Au}_{n} \mathrm{O}_{2}\right], \\
& E_{b 2}=E\left[\mathrm{Au}_{n} \mathrm{O}_{2}\right]+E\left[\mathrm{O}_{2}\right]-E\left[\mathrm{Au}_{n}\left(\mathrm{O}_{2}\right)_{2}\right] .
\end{aligned}
$$

Here $n$ is the number of atoms in the gold cluster and $E[X]$ is the energy of the ground electronic state of the molecule $X$. The binding energies of the oxygen molecules to the negatively charged gold clusters were determined in the same way. Positive values for $E_{b 1}$ and $E_{b 2}$ indicate that adding the oxygen molecule lowers the energy. A negative value indicates that the energy of the oxygenated cluster (although a minimum on the PES) is higher than that of the separated reactants.

$\mathrm{Au}_{2} \mathrm{O}_{2}$ and $\mathrm{Au}_{2}\left(\mathrm{O}_{2}\right)_{2}$ are unstable at both the MP2 and HF levels of theory. In contrast, the previous DFT/PW91 calculation ${ }^{2}$ predicts a binding energy of $0.49 \mathrm{eV}$ for the first oxygen molecule and $0.29 \mathrm{eV}$ for the second. There is thus a substantial difference between the DFT and MP2 results.

\section{E. Results for $\mathrm{Au}_{2} \mathrm{O}_{2}^{-}$and $\mathrm{Au}_{2}\left(\mathrm{O}_{2}\right)_{2}^{-}$}

As shown in Table $\mathrm{V}, \operatorname{CCSD}(\mathrm{T})$ theory gives $1.07 \mathrm{eV}$ for the binding energy of $\mathrm{O}_{2}$ to $\mathrm{Au}_{2}^{-}$. This is slightly lower than the value predicted by the B3LYP calculations performed in the present work $(1.22 \mathrm{eV})$, the plane wave PW91 DFT calculations $(1.40 \mathrm{eV}),{ }^{2}$ and the plane wave PBE DFT calculations $(1.39 \mathrm{eV}) .{ }^{4(\mathrm{a})}$ The experiments of Lee and $\mathrm{Ervin}^{24}$ give $1.01 \pm 0.14 \mathrm{eV}$. Again, the binding energy predicted by density functional theory is too high. This is not entirely surprising, since it has been noted frequently that DFT tends to "overbind." ${ }^{25}$ If this error were systematic, one could "correct" for it and bring the results closer to reality. Unfortunately, as discussed in the following, the errors do not appear to be systematic overbinding.

The CCSD $(\mathrm{T})$ binding energy of the second $\mathrm{O}_{2}$ to form $\mathrm{Au}_{2}\left(\mathrm{O}_{2}\right)_{2}^{-}$is $-0.56 \mathrm{eV}$. The negative value indicates that this local minimum on the potential energy surface is higher in energy than the sum of the energies of the separated $\mathrm{O}_{2}$ $+\mathrm{Au}_{2} \mathrm{O}_{2}^{-}$. This implies the presence of an intervening barrier for the removal of the second $\mathrm{O}_{2}$ molecule from the $\mathrm{Au}_{2}$ cluster. MP2 predicts a negative $E_{2 b}$ that is even larger in

TABLE IV. Bond distances $R(\AA)$, angles $\Theta(\mathrm{deg})$, and energies $\Delta E(\mathrm{kcal} / \mathrm{mol})$ relative to $\mathrm{Au}_{3}^{-1} \Sigma_{g}^{+}$state at

\begin{tabular}{|c|c|c|c|c|c|c|}
\hline & \multirow{2}{*}{$\begin{array}{c}{ }^{1} \Sigma_{g}^{+} \\
R\end{array}$} & \multicolumn{3}{|c|}{$C_{2 v}\left({ }^{1} A_{1}\right)$} & \multicolumn{2}{|c|}{$D_{3 h}\left({ }^{3} A_{1}\right)$} \\
\hline & & $\Delta E$ & $R$ & $\Theta$ & $\Delta E$ & $R$ \\
\hline $\mathrm{HF}$ & 2.711 & 37.1 & 3.326 & 46.2 & 18.8 & 2.880 \\
\hline MP2 & 2.550 & 37.8 & 2.785 & 53.6 & 23.4 & 2.664 \\
\hline $\operatorname{CCSD}(\mathrm{T})$ & $\mathrm{MP} 2^{\mathrm{b}}$ & 36.0 & MP2 & MP2 & 24.4 & MP2 \\
\hline $\operatorname{CASSCF}(3,4)$ & 2.718 & 40.5 & MP2 & MP2 & 24.2 & 2.880 \\
\hline MRMP2 & 2.559 & 38.8 & MP2 & MP2 & 18.4 & MP2 \\
\hline B3LYP & 2.631 & 38.9 & 2.945 & 52.2 & 28.4 & 2.78 \\
\hline $\mathrm{R}-\operatorname{CCSD}(\mathrm{T})^{\mathrm{a}}$ & 2.573 & 41.3 & 2.844 & 53.4 & 29.1 & 2.717 \\
\hline R-MP2 ${ }^{\mathrm{a}}$ & 2.511 & 45.4 & 2.792 & 52.7 & 30.0 & 2.647 \\
\hline
\end{tabular}
different levels of theory.

${ }^{a}$ Relativistic $\operatorname{CCSD}(\mathrm{T})$ and MP2 with all electron $(10 s 7 p 5 d 3 f) /[10 s 6 p 5 d 3 f]$ basis set (Ref. 21).

${ }^{\mathrm{b}} \mathrm{MP} 2$ geometries. 

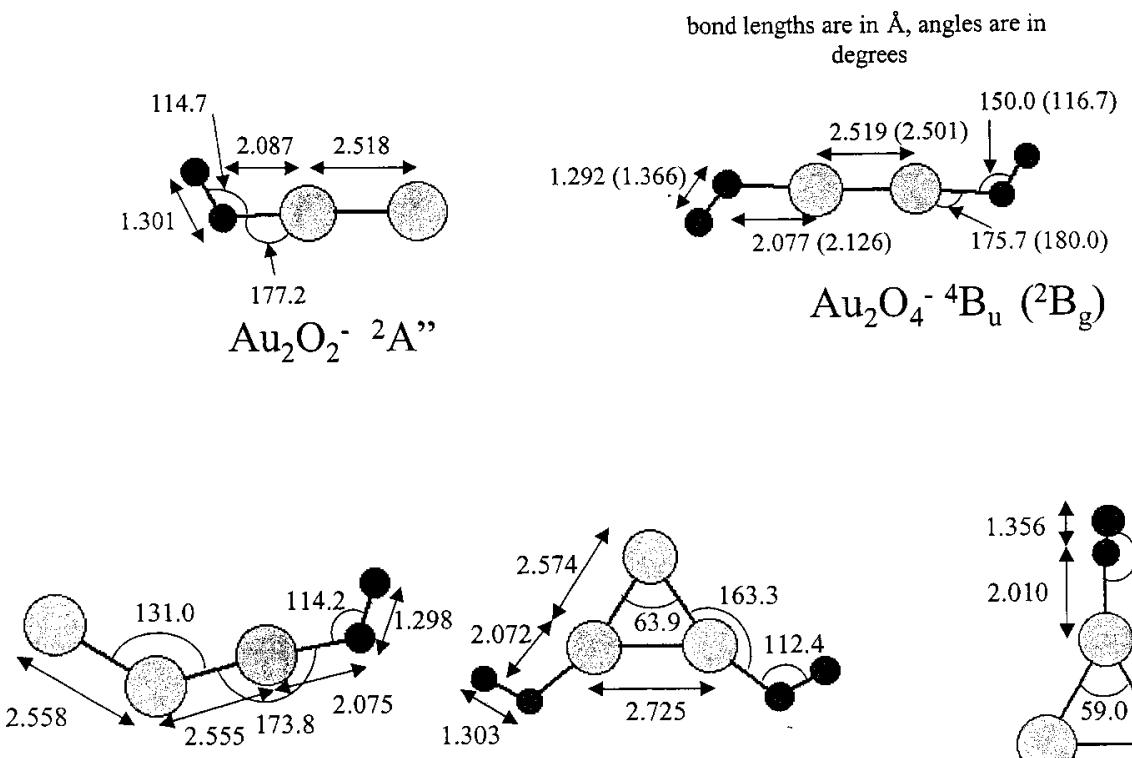

$\mathrm{Au}_{3} \mathrm{O}_{2}{ }^{-3} \mathrm{~A}^{\prime \prime}$

$\mathrm{Au}_{3} \mathrm{O}_{4}^{-{ }^{3} \mathrm{~B}_{1}}$

magnitude. Plane wave DFT/PW91 calculations give a binding energy, $E_{2 b}=0.71 \mathrm{eV}$, that is opposite in sign to the $\operatorname{CCSD}(\mathrm{T})$ and MP2 results. The discrepancy between the MP2 and CCSD(T), on the one hand, and the DFT calculation on the other, is substantial and again suggests that this level of theory tends to severely overbind oxygen to Au clusters.

Salisbury, Wallace, and Whetten ${ }^{26}$ found that negatively charged Au clusters (a) will not adsorb $\mathrm{O}_{2}$ if the cluster has an even number of electrons, and (b) will adsorb one $\mathrm{O}_{2}$ molecule, but not a second one, if the cluster has an odd number of electrons. The MP2 and $\operatorname{CCSD}(\mathrm{T})$ results are in agreement with these experimental results, while the planewave DFT/PW91 results are not. The fast-flow reactor method used by Salisbury et al. has some limitations, as discussed recently by Wallace, Leavitt, and Whetten. ${ }^{27}$ However, the experimental results taken together with the MP2 and $\operatorname{CCSD}(\mathrm{T})$ calculations provide reasonable evidence that DFT does not describe the $\mathrm{Au}_{2}\left(\mathrm{O}_{2}\right)_{2}^{-}$complex correctly.

Figure 3 illustrates the manner in which the electronic states of $\mathrm{Au}_{2}^{-}$and two $\mathrm{O}_{2}$ molecules may correlate with the electronic states of $\mathrm{Au}_{2} \mathrm{O}_{2}^{-}$and $\mathrm{Au}_{2}\left(\mathrm{O}_{2}\right)_{2}^{-}$. The ground state of $\mathrm{Au}_{2}\left(\mathrm{O}_{2}\right)_{2}^{-}$is a quartet, whereas the ground states of $\mathrm{Au}_{2}^{-}$ and $\mathrm{Au}_{2} \mathrm{O}_{2}^{-}$are doublets, and that of $\mathrm{O}_{2}$ is a triplet. Therefore, $\mathrm{Au}_{2}^{-}$and $\mathrm{O}_{2}$ can spin couple to produce either a doublet or a quartet. The present calculations, as noted earlier, predict that the doublet is the ground state. So, there is no crossing

TABLE V. $\mathrm{Au}_{2}^{-}$. Binding energies $(\mathrm{eV})$ for the first, $E_{b 1}$, and the second, $E_{b 2}, \mathrm{O}_{2}$.

\begin{tabular}{lrrrcc}
\hline \hline & HF & MP2 & CCSD(T) & B3LYP & PW-PW91 $^{\mathrm{a}}$ \\
\hline$E_{b 1}$ & 0.69 & 0.51 & 1.07 & 1.22 & 1.40 \\
$E_{b 2}$ & -0.52 & -1.57 & -0.56 & $\mathrm{~b}$ & 0.71 \\
\hline \hline
\end{tabular}

${ }^{\mathrm{a} D F T}$ with plane-wave basis set and PW91 functional (Ref. 2).

${ }^{\mathrm{b}}$ The B3LYP calculation for $E_{b 2}$ did not converge.

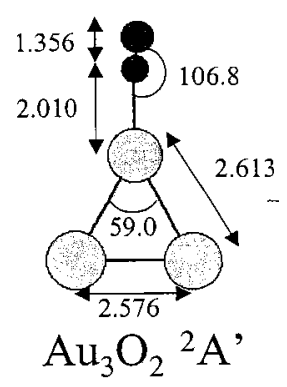

FIG. 2. MP2 optimized geometries of the lowest electronic states of $\mathrm{Au}_{2} \mathrm{O}_{2}^{-}$, $\mathrm{Au}_{2}\left(\mathrm{O}_{2}\right)_{2}^{-}, \mathrm{Au}_{3} \mathrm{O}_{2}^{-}$, and $\mathrm{Au}_{3}\left(\mathrm{O}_{2}\right)_{2}^{-}$, and $\mathrm{Au}_{3} \mathrm{O}_{2}$ complexes. For $\mathrm{Au}_{2}\left(\mathrm{O}_{2}\right)_{2}^{-}$, the geometries of the lowest quartet and doublet states are shown. of the doublet and quartet potential energy surfaces as the first oxygen molecule is added to the $\mathrm{Au}_{2}$ cluster. Adding the second $\mathrm{O}_{2}$ to the ground doublet state of $\mathrm{Au}_{2} \mathrm{O}_{2}^{-}$can again produce either a doublet or a quartet. In this case, the calculations predict that the ground state of the product $\mathrm{Au}_{2}\left(\mathrm{O}_{2}\right)_{2}^{-}$ is the quartet. So, at some point between $\mathrm{Au}_{2} \mathrm{O}_{2}^{-}+\mathrm{O}_{2}$ and $\mathrm{Au}_{2}\left(\mathrm{O}_{2}\right)_{2}^{-}$, the doublet and quartet potential energy surfaces cross. While an exhaustive study of these potential energy surfaces is beyond the scope of the present work, these results suggest that nonadiabatic interactions, such as spinorbit coupling, could be important in this region of the potential energy surfaces.

The fact that the energy of $\mathrm{Au}_{2}\left(\mathrm{O}_{2}\right)_{2}^{-}$is higher than that of $\mathrm{Au}_{2}\left(\mathrm{O}_{2}\right)_{2}^{-}+\mathrm{O}_{2}$ indicates that at equilibrium the probability of observing this cluster with two oxygen molecules on it is small. This is consistent with the experiments of Salisbury et al., assuming the clusters are in thermal equilibrium in their experiment. The results of the DFT/PW91 calculations, according to which the second oxygen binds strongly, are compatible with the experiments only if it is valid to assume that the rate of the reaction $\mathrm{Au}_{2} \mathrm{O}_{2}^{-}+\mathrm{O}_{2} \rightarrow \mathrm{Au}_{2}\left(\mathrm{O}_{2}\right)_{2}^{-}$is so

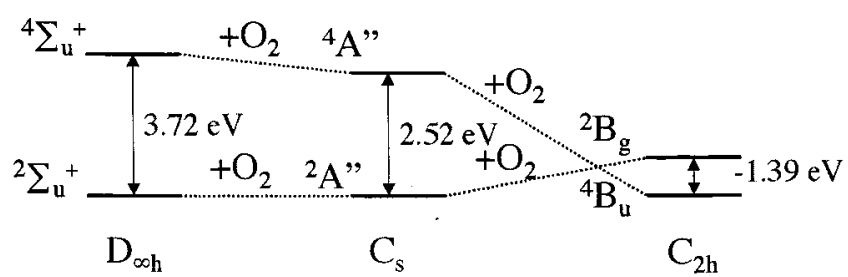

$$
\mathrm{Au}_{2}^{-}+\mathrm{O}_{2}-->\mathrm{Au}_{2} \mathrm{O}_{2}^{-}+\mathrm{O}_{2}-->\mathrm{Au}_{2}\left(\mathrm{O}_{2}\right)_{2}^{-}
$$

Energies were calculated at $\operatorname{CCSD}(\mathrm{T})$ level of theory

FIG. 3. Correlation of the electronic states of $\mathrm{Au}_{2}^{-}$and two $\mathrm{O}_{2}$ molecules with the electronic states of $\mathrm{Au}_{2} \mathrm{O}_{2}^{-}$and $\mathrm{Au}_{2}\left(\mathrm{O}_{2}\right)_{2}^{-}$. 
TABLE VI. $\mathrm{Au}_{3} / \mathrm{Au}_{3}^{-}$. Binding energies (eV) for the first, $E_{b 1}$, and the second, $E_{b 2}, \mathrm{O}_{2}$. Unstable means that the molecule does not bind. Because of this $\operatorname{CCSD}(\mathrm{T})$ cannot be performed. B3LYP converged only for $\mathrm{Au}_{3}$, $E_{b 1}$.

\begin{tabular}{lrrrcc}
\hline \hline & \multicolumn{1}{c}{ HF } & MP2 & CCSD(T) & B3LYP & PW-PW91 $^{\text {a }}$ \\
\hline $\mathrm{Au}_{3}^{-}, E_{b 1}$ & -0.38 & -1.10 & -0.38 & & 0.37 \\
$\mathrm{Au}_{3}^{-}, E_{b 2}$ & 0.57 & 0.52 & 1.07 & & 0.70 \\
$\mathrm{Au}_{3}, E_{b 1}$ & -0.14 & -0.68 & 0.08 & 0.19 & 0.90 \\
$\mathrm{Au}_{3}, E_{b 2}$ & Unstable & Unstable & & & 0.59 \\
\hline \hline
\end{tabular}

${ }^{\mathrm{a} D F T}$ with plane-wave basis set and PW91 functional (Ref. 2).

low that $\mathrm{Au}_{2}\left(\mathrm{O}_{2}\right)_{2}^{-}$is not formed during the experiments. However, the most plausible conclusion is that the DFT calculations are in error.

These results for $\mathrm{Au}_{2}\left(\mathrm{O}_{2}\right)_{2}^{-}$are relevant to the mechanism for $\mathrm{CO}$ oxidation by a $\mathrm{Au}_{2}^{-}$cluster proposed by Hakkinen and Landman. ${ }^{4(a)}$ Based on DFT calculations, they proposed that catalysis takes place by adsorption of $\mathrm{O}_{2}$ and $\mathrm{CO}$ to form a carbonate. These authors did not consider the possibility that a second oxygen molecule may adsorb on the cluster, blocking CO adsorption. The DFT/PW91 calculations, ${ }^{2}$ which give (probably incorrectly) the binding energy for the second oxygen to be $0.71 \mathrm{eV}$, indicate that the rates of adsorption and desorption of the second oxygen ought to be included as one of the steps in the kinetic scheme. The present MP2 and $\operatorname{CCSD}(\mathrm{T})$ calculations show, however, that $\mathrm{Au}_{2}\left(\mathrm{O}_{2}\right)_{2}^{-}$is an unstable local minimum, so its formation will not affect $\mathrm{CO}$ oxidation. Another rationale for ignoring the adsorption of a second oxygen molecule in the $\mathrm{CO}$ oxidation process is the large predicted adsorption energy of $\mathrm{CO}^{4(\mathrm{a})}$

\section{F. Oxygen binding to $\mathrm{Au}_{3}$}

The binding energies of one or two oxygen molecules to the $\mathrm{Au}_{3}$ cluster are given in Table VI. $\operatorname{CCSD}(\mathrm{T})$ predicts the first oxygen molecule barely binds to $\mathrm{Au}_{3}\left(E_{b 1}=0.08 \mathrm{eV}\right)$ while DFT/PW91 predicts a binding energy of $0.90 \mathrm{eV} .^{2}$ This degree of overbinding predicted by DFT is too large to be considered modest or systematically correctable. Both MP2 and HF predict a negative value for $E_{b 1}$, while B3LYP predicts $+0.19 \mathrm{eV}$. It is interesting that in this case the Gaussian-based hybrid functional is in considerably better agreement with the benchmark $\operatorname{CCSD}(\mathrm{T})$ calculations than is the plane wave-based PW91 approach. Mills, Gordon, and Metiu $^{2}$ extended, to neutral gold clusters, the rules proposed by Salisbury et $a .^{26}$ for adsorption of oxygen on negatively charged gold clusters. This extension, which was supported by the prior plane wave DFT/PW91 results, ${ }^{2}$ postulates that oxygen binds weakly to neutral Au clusters having an even number of electrons, and more strongly to those having an odd number of electrons. The present $\operatorname{CCSD}(\mathrm{T})$ results are clearly incompatible with this conjecture.

The PW91 calculations predict that the binding energy of the second oxygen molecule to $\mathrm{Au}_{3}$ is fairly strong (0.59 $\mathrm{eV})$, whereas $\operatorname{CCSD}(\mathrm{T})$ predicts no binding. Clearly, the DFT/PW91 predictions for oxygen binding to $\mathrm{Au}_{3}$ are in strong disagreement with those of $\operatorname{CCSD}(\mathrm{T})$.

\section{G. Oxygen binding to $\mathrm{Au}_{3}^{-}$}

As shown in Table VI, for $\mathrm{Au}_{3} \mathrm{O}_{2}^{-}, \mathrm{CCSD}(\mathrm{T})$ predicts a bound state whose energy exceeds that of the separated $\mathrm{Au}_{3}^{-}$ and $\mathrm{O}_{2}$, in their ground state, by $0.38 \mathrm{eV}$; that is, $E_{b 1}$ is negative. MP2 predicts the binding energy to be even more negative. In contrast, DFT/PW91 calculation finds that the energy of the bound state of $\mathrm{Au}_{3} \mathrm{O}_{2}^{-}$is $0.37 \mathrm{eV}$ below that of the fragments; that is, $E_{b}$ is predicted to be positive. Therefore, the rule proposed by Salisbury et al. ${ }^{26}$ that oxygen does not bind to the cluster $\mathrm{Au}_{3}^{-}$, because it has an even number of electrons, is in agreement with the $\operatorname{CCSD}(\mathrm{T})$ and MP2 results. The DFT calculation is in quantitative disagreement with this rule, since it indicates binding. However, DFT/PW91 does predict that the binding of one $\mathrm{O}_{2}$ molecule to $\mathrm{Au}_{3}^{-}$is much weaker than the binding to $\mathrm{Au}_{2}^{-}$, in qualitative agreement with the rule of Salisbury et al.

Of the compounds studied here, $\mathrm{Au}_{3}\left(\mathrm{O}_{2}\right)_{2}^{-}$is the most surprising: the $\operatorname{CCSD}(\mathrm{T})$ binding energy of the second oxygen, $E_{b 2}$, is $1.07 \mathrm{eV}$. This means that the energy

$$
E_{b 2}+E_{b 1}=-E\left(\mathrm{Au}_{3}\left(\mathrm{O}_{2}\right)_{2}^{-}\right)+E\left(\mathrm{Au}_{3}^{-}\right)+2 E\left(\mathrm{O}_{2}\right)
$$

for forming $\mathrm{Au}_{3}\left(\mathrm{O}_{2}\right)_{2}^{-}$from oxygen and $\mathrm{Au}_{3}^{-}$, is 1.07 $+(-0.38)=0.69$; that is, the complex is stable with respect to separated $2 \mathrm{O}_{2}$ and $\mathrm{Au}_{3}^{-}$.

This result seems to imply that the complex $\mathrm{Au}_{3}\left(\mathrm{O}_{2}\right)_{2}^{-}$ should be observed in the experiments, even though it is not seen. However, the absence of the complex in the experiments may be rationalized as follows. At the pressures used in the experiments, three-body collisions of $\mathrm{Au}_{3}^{-}$with two oxygen molecules are very unlikely. Therefore, the complex must be formed by successive adsorption of oxygen molecules. However, the probability of forming $\mathrm{Au}_{3} \mathrm{O}_{2}^{-}$is extremely low, since its energy exceeds that of separated $\mathrm{Au}_{3}^{-}$ and $\mathrm{O}_{2}$. For this reason the rate of forming $\mathrm{Au}_{3}\left(\mathrm{O}_{2}\right)_{2}^{-}$ should be small. Of course, the fact that $\mathrm{Au}_{3}\left(\mathrm{O}_{2}\right)_{2}^{-}$is bound relative to its separated constituents means that it must be present in an equilibrium mixture. However, the kinetics indicates that the time to reach equilibrium must be much longer than that used in the experiments of Salisbury et al. ${ }^{26}$ Therefore, the absence of $\mathrm{Au}_{3}\left(\mathrm{O}_{2}\right)_{2}^{-}$in the experiments is not in conflict with the $\operatorname{CCSD}(\mathrm{T})$ results. It does, however, disagree with the DFT/PW91 method, which predicts that clusters with one and two oxygen atoms on them should be observed. Although the experimental error is unknown, the $\operatorname{CCSD}(\mathrm{T})$ results are consistent with the available experimental evidence, and this is unlikely to change after a rigorous error analysis of the experiments is performed.

\section{SUMMARY}

It is assumed that the $\operatorname{CCSD}(\mathrm{T})$ calculations performed here represent the current state of the art, against which the other levels of theory may be measured, especially when experimental data are lacking. Therefore, one concludes that density functional theory works well for the small neutral or negatively charged Au clusters considered here. In particular, DFT gives good results for the binding energy of $\mathrm{Au}_{2}$, the electron affinity of $\mathrm{Au}_{2}$, and the geometries of the larger clusters. However, DFT fails badly when it calculates the 
binding energies of one or two oxygen molecules to $\mathrm{Au}_{2}$, $\mathrm{Au}_{2}^{-}, \mathrm{Au}_{3}$, and $\mathrm{Au}_{3}^{-}$. One may argue that it is more difficult to correctly describe the small clusters than the larger ones, and that the DFT results become better as the number of $\mathrm{Au}$ atoms is increased. However, the failure appears to be connected to the $\mathrm{O}_{2}$ molecule, which is notoriously difficult to describe correctly by quantum chemical calculations. Binding of $\mathrm{O}_{2}$ to $\mathrm{Au}_{n}$ involves a substantial charge transfer, and describing an $\mathrm{O}_{2}$ molecule with an excess of electronic charge is even more difficult. There is a chance that binding of other molecules to $\mathrm{Au}$ is less demanding. Calculations on the binding of $\mathrm{H}_{2}$ molecules to neutral and anionic Au clusters are now in progress.

The results presented here do not affect the mechanism proposed by Hakkinen and Landman for $\mathrm{CO}$ oxidation by $\mathrm{Au}_{2}^{-}$. While the binding energy $(1.07 \mathrm{eV})$ of $\mathrm{O}_{2}$ to $\mathrm{Au}_{2}^{-}$ found by $\operatorname{CCSD}(\mathrm{T})$ differs from that found by Hakkinen and Landman $(1.39 \mathrm{eV})$, this difference does not affect the qualitative conclusions drawn in their paper. Of course, only one step in their mechanism has been examined here.

It does not appear that the deviations of DFT from the $\operatorname{CCSD}(\mathrm{T})$ results are either systematic or predictable by some simple rule. Unfortunately, this means that no guidance can be provided regarding some reasonable corrections to be made when one uses DFT to study oxidation reactions.

The discrepancy between DFT and $\operatorname{CCSD}(\mathrm{T})$ is particularly dramatic because oxygen is involved. In a large number of cases, DFT calculations agree well with experiments. ${ }^{25}$ We have also found that the results of PW91 DFT and those of B3LYP agree well with the $\operatorname{CCSD}(\mathrm{T})$ results for $\mathrm{H}$ adsorbed on Au clusters. ${ }^{30}$ Moreover, B3LYP calculations in Michael Bowers's group ${ }^{31}$ agree well with the binding energies of oxygen to small positive Ag clusters. In this case the binding energy of $\mathrm{O}_{2}$ is small and the oxygen molecule is not changed much upon binding, resulting in cancellation of the errors made on oxygen molecules. Because the behavior of DFT is not predictable, it is nevertheless important that cases of failure are carefully cataloged and documented along with the successes, to serve as a target for new and more accurate versions of DFT and to warn the users that in certain systems the DFT results should be used with caution.

\section{ACKNOWLEDGMENTS}

The authors thank Dr. Michael Schmidt, Dr. Greg Mills, and Dr. Dmitri Fedorov for helpful discussions. This work was supported by AFOSR through a DURINT grant. The calculations in this work were performed in part on an IBM workstation cluster made possible by grants from IBM in the form of a Shared University Research grant and the United States Department of Energy.
${ }^{1}$ M. Haruta, Catal. Today 36, 153 (1997); M. Haruta and M. Date, Appl. Catal., A 222, 427 (2001); G. Bond and D. T. Thomson, Catal. Rev. Sci. Eng. 41, 319 (1999), G. C. Bond, Catal. Today 72, 5 (2002).

${ }^{2}$ G. Mills, M. S. Gordon, and H. Metiu, Chem. Phys. Lett. 359, 493 (2002).

${ }^{3}$ A. Sanchez, S. Abbet, U. Heiz, W. D. Shcneider, H. Hakkinen, R. N. Barnett, and U. Landman, J. Phys. Chem. A 103, 9573 (1999).

${ }^{4}$ (a) H. Hakkinen and U. Landman, J. Am. Chem. Soc. 123, 9704 (2001); (b) N. Lopez and J. K. Norskov, ibid. 124, 11262 (2002); (c) Z.-P. Liu, P. Hu, and A. Alavi, ibid. 124, 14770 (2002).

${ }^{5}$ C. Hampel and H.-J. Werner, J. Chem. Phys. 104, 6286 (1996); G. Hetzer, P. Pulay, and H.-J. Werner, Chem. Phys. Lett. 290, 143 (1998); M. Schütz, G. Hetzer, and H.-J. Werner, J. Chem. Phys. 111, 5691 (1999).

${ }^{6}$ A. D. Becke, J. Chem. Phys. 98, 5648 (1993); P. J. Stephens, F. J. Devlin, C. F. Chablowski, and M. J. Frisch, J. Phys. Chem. 98, 11623 (1994); R. H. Hertwig and W. Koch, Chem. Phys. Lett. 268, 345 (1997).

${ }^{7}$ Y. Wang and J. P. Perdew, Phys. Rev. B 44, 13298 (1991).

${ }^{8}$ C. Möller and M. S. Plesset, Phys. Rev. 46, 618 (1934).

${ }^{9}$ R. J. Bartlett, J. Phys. Chem. 93, 1697 (1989); C. Hampel, K. Peterson, and H.-J. Werner, Chem. Phys. Lett. 190, 1 (1992), and references therein. The program to compute the perturbative triples corrections has been developed by M. J. O. Deegan and P. J. Knowles (1992).

${ }^{10}$ T. J. Lee and D. Jayatilaka, Chem. Phys. Lett. 201, 1 (1993); T. J. Lee, A. P. Rendell, K. G. Dyall, and D. Jayatilaka, J. Chem. Phys. 100, 7400 (1994).

${ }^{11}$ P. J. Knowles, C. Hampel, and H.-J. Werner, J. Chem. Phys. 99, 5219 (1993).

${ }^{12}$ B. O. Roos, Adv. Chem. Phys. 69, 399 (1987).

${ }^{13}$ K. Hirao, Chem. Phys. Lett. 190, 374 (1992).

${ }^{14}$ W. J. Stevens, M. Krauss, H. Basch, and P. G. Jasien, Can. J. Chem. 70, 612 (1992).

${ }^{15}$ M. W. Schmidt, K. K. Baldridge, J. A. Boatz et al., J. Comput. Chem. 14, 1347 (1993); G. D. Fletcher, M. W. Schmidt, and M. S. Gordon, Adv. Chem. Phys. 110, 267 (1999); G. D. Fletcher, M. W. Schmidt, B. M. Bode, and M. S. Gordon, Comput. Phys. Commun. 128, 190 (2000).

${ }^{16}$ MOLPRO is a package of $a b$ initio programs written by H.-J. Werner and P. J. Knowles, with contributions from R. D. Amos, A. Bernhardsson, A. Berning et al.

${ }^{17}$ J. Ho, K. M. Ervin, and W. C. Lineberger, J. Chem. Phys. 93, 6987 (1990); G. F. Gantefor, D. M. Cox, and A. Kaldor, ibid. 93, 8395 (1990).

${ }^{18}$ K. J. Taylor, C. L. Pettiette-Hall, O. Chesnovsky, and R. E. Smalley, J. Chem. Phys. 96, 3319 (1992)

${ }^{19}$ J. P. Perdew, K. Burke, and M. Ernzerhof, Phys. Rev. Lett. 77, 3865 (1996).

${ }^{20}$ J. A. Howard, R. Sutcliffe, and B. Mille, J. Chem. Soc., Chem. Commun. 1983, 1449; J. A. Howard, R. Sutcliffe, and B. Mille, Surf. Sci. 156, 214 (1985).

${ }^{21}$ R. Wesendrup, T. Hunt, and P. Schwerdtfeger, J. Chem. Phys. 112, 9356 (2000).

${ }^{22}$ H.-J. Werner and P. J. Knowles, J. Chem. Phys. 89, 5803 (1988); P. J. Knowles and H.-J. Werner, Chem. Phys. Lett. 145, 514 (1988).

${ }^{23}$ (a) K. Balasubramanian and M. Z. Liao, Chem. Phys. 127, 313 (1988); (b) K. Balasubramanian and K. K. Das, Chem. Phys. Lett. 186, 577 (1991).

${ }^{24}$ T. H. Lee, Ph.D. thesis, University of Nevada, Reno, 1995.

${ }^{25}$ (a) M. Scheffler and C. Stampfl, in Handbook of Surface Science, edited by K. Horn and M. Scheffler (Elsevier, Amsterdam, 1999); (b) B. Hammer and J. K. Norskov, Adv. Catal. 45, 71 (2000).

${ }^{26}$ B. E. Salisbury, W. T. Wallace, and R. L. Whetten, Chem. Phys. 262, 131 (2000).

${ }^{27}$ W. T. Wallace, A. J. Leavitt, and R. L. Whetten, Chem. Phys. Lett. (to be published).).

${ }^{28}$ B. A. Hess and U. Kaldor, J. Chem. Phys. 112, 1809 (2000).

${ }^{29}$ K. P. Huber and G. Herzberg, Molecular Spectra and Molecular Structure. IV. Constants of Diatomic Molecules (Van Nostrand, New York, 1979).

${ }^{30}$ S. A. Varganov, R. M. Olson, M. S. Gordon, and H. Metiu (unpublished).

${ }^{31}$ M. Manard and M. Bowers (private communication). 